Int. J. Anal. Appl. (2022), 20:3

\title{
International Journal of Analysis and Applications
}

\section{Well-Posedness of Triequilibrium-Like Problems}

\author{
Misbah Iram Bloach, Muhammad Aslam Noor*, Khalida Inayat Noor \\ Department of Mathematics, COMSATS University Islamabad, Islamabad, Pakistan \\ *Corresponding author: noormaslam@gmail.com
}

\begin{abstract}
This work emphasizes in presenting new class of equilibrium-like problems, termed as equilibriumlike problems with trifunction. We establish some metric characterizations for the well-posed triequilibriumlike problems. We give some conditions under which the equilibrium-like problems are strongly wellposed. Our results, which give essential and adequate conditions to the well-posedness of triequilibriumlike problems, are acquired by utilizing the assumption of pseudomonotonicity. Technique and ideas of this paper inspire further research in this dynamic field.
\end{abstract}

\section{Introduction}

The theory of equilibrium problems is an engrossing and significant offshoot of variational inequalities in practice with a broad variety of industrial, physical, geographical and social applications. Across various areas of pure, applied and engineering sciences, equilibrium problem theory has shown incredible potential and great impact. In nearly all disciplines, of mathematics and engineering, this theory has registered its exceptional ever-expanding mark. A new and incisive treatment of a broad list of problems, that occur in ecology, finance, economics, elasticity, network, image reconstruction, optimization and transport, are exhaustively approached by the equilibrium problem theory. In 1994, Blum and Oettli [3] and Noor and Oettli [23] rendered equilibrium problems their existing form. The classical equilibrium problems theory revolves around the assumption of convexity of the set and objective function. Equilibrium problems cover a diverse set of applications including hemivariational inequalities, variational inequalities, game theory, Nash equilibrium, variational-like inequalities and fixed point

Received: Sep. 08, 2021.

2010 Mathematics Subject Classification. 49J40, 90C33.

Key words and phrases. variational-like inequalities; preinvex functions; equilibrium problems; well-posed; pseudomonotonicity. 
point theory, see $[1-7,12-19,21,22,24-29]$.

Recently, the notion of convexity has started expanding to numerous fields showing the capacity for various useful applications. Hanson [8] derived invex functions as a special extension of convex functions. Different results were presented to make this fact noticeable that what holds for convex functions in mathematical programming also holds for a generalized class of functions known as invex functions. Ben-Israel and Mond [1] and Weir and Jeyakumar [31] works led to preinvex functions as another generalized class of convex functions. Weir and Mond [30] has shown in their work the interchangeability of preinvex functions with convex functions in optimization problems. In [15], it is made evident that the minimum of preinvex on the invex set can be disciplined into variational inequalities, widely known as variational-like inequalities. Variational-like inequalities and equilibrium-like problems, owing to their specialized nature, cannot allow traditional resolvent method, projection method and their prevalent variant forms to propound any iterative methods. To bridge this gap, we resort to a technique named as auxiliary principle, proposed by Glowinski et al. [4]. To solve numerous variational inequalities and equilibrium problems, Noor $[15,19,20]$ and Noor et al. $[22,24,25,27]$ employed the technique of auxiliary principle to propose various iterative methods.

Research of our paper is devoted to present a new class of equilibrium problems, termed as equilibrium-like problems with trifunction. The notion of well-posedness of variational ineaqualities and equilibrium problems was introduced by Lucchetti and Patrone $[9,10]$. We expand the notion of well-posedness to contemplate and establish the well-posedness of triequilibrium-like problems. Several interesting and important cases are discussed as applications of the obtained results.

\section{Basic concepts and formulations}

Let $\tilde{H}$ be a real Hilbert space. The inner product and norm on $\tilde{H}$ are denoted by $\langle.,$.$\rangle and \|$. $\|$ respectively. Let $\mathbb{K}_{\hbar}$ be a nonempty invex set in $\tilde{H}$. Let $\Im: \mathbb{K}_{\hbar} \rightarrow \Re$ and $\hbar(.,):. \tilde{H} \times \tilde{H} \rightarrow \tilde{H}$ are continuous functions.

First we recall the following well-known results and concepts, before discussing our main results.

Definition 2.1. ([1]). A nonempty set $\mathbb{K}_{\hbar}$ in $\tilde{H}$ is said to be an invex set, if there exists a bifunction $\hbar(.,$.$) , such that$

$$
\xi+\tau \hbar(\zeta, \xi) \in \mathbb{K}_{\hbar}, \forall \xi, \zeta \in \mathbb{K}_{\hbar}, \tau \in[0,1] .
$$

If the set $\mathbb{K}_{\hbar}$ is invex at each $\xi \in \mathbb{K}_{\hbar}$, then $\mathbb{K}_{\hbar}$ is also called $\hbar$-connected set.

Definition 2.2. ([1]). A function $\Im: \mathbb{K}_{\hbar} \rightarrow \Re$ is said to be a preinvex function, if there exists a bifunction $\hbar(.,$.$) , such that$

$$
\Im(\xi+\tau \hbar(\zeta, \xi)) \leq(1-\tau) \Im(\xi)+\tau \Im(\zeta), \forall \xi, \zeta \in \mathbb{K}_{\hbar}, \tau \in[0,1]
$$


The function $\Im: \mathbb{K}_{\hbar} \rightarrow \Re$ is said to be preconcave if and only if $-\Im$ is preinvex.

Definition 2.3. ([8]). A differentiable function $\Im: \mathbb{K}_{\hbar} \rightarrow \Re$ is said to be an invex function, if there exists a bifunction $\hbar(.,$.$) , such that$

$$
\Im(\zeta)-\Im(\xi) \geq\left\langle\Im^{\prime}(\xi), \hbar(\zeta, \xi)\right\rangle, \forall \xi, \zeta \in \mathbb{K}_{\hbar}, \tau \in[0,1],
$$

where $\Im^{\prime}(\xi)$ is the differential of $\Im$ at $\xi$. From above definitions, it is clear that the differentiable preinvex functions are the invex functions but the converse is not true, see [21]. We note that, if $\hbar(\zeta, \xi)=\zeta-\xi$, the invex set $\mathbb{K}_{\hbar}$ reduces to the convex set $\mathbb{K}$ and preinvex functions reduce to convex functions. There are some functions which are preinvex but not convex.

Definition 2.4. . The bifunction $\hbar(.,):. \tilde{H} \times \tilde{H} \rightarrow \Re$ satisfies the following condition

$$
\begin{array}{r}
\hbar\left(\xi+\tau_{1}(\zeta-\xi), \xi+\tau_{2}(\zeta-\xi)\right)=\left(\tau_{1}-\tau_{2}\right) \hbar(\zeta, \xi) \\
\forall \xi, \zeta \in \tilde{H} .
\end{array}
$$

For $\tau_{1}=0,1$ and $\tau_{2}=\tau$, we get Condition C of Mohan and Neogy [11],

(i) $\hbar(\xi, \xi+\tau(\zeta-\xi))=-\tau \hbar(\zeta, \xi)$,

(ii) $\hbar(\zeta, \xi+\tau(\zeta-\xi))=(1-\tau) \hbar(\zeta, \xi), \forall \xi, \zeta \in \tilde{H}$.

Mohan and Neogy [11] used definition 2.4 to show that an invex function on an invex set $\mathbb{K}_{\hbar}$, is also a preinvex function and the converse also hold.

Given an operator $\Upsilon: \tilde{H} \rightarrow \Re$ and a continuous trifunction $\Psi(., \ldots): \tilde{H} \times \tilde{H} \times \tilde{H} \rightarrow \Re$, consider the problem of finding $\xi \in \mathbb{K}_{\hbar}$, such that

$$
\Psi(\xi, \Upsilon(\xi), \hbar(\zeta, \xi)) \geq 0, \forall \zeta \in \mathbb{K}_{\hbar}
$$

The problem (2.1) is called an equilibrium-like problem with trifunction.

For $\Psi(\xi, \Upsilon(\xi), \hbar(\zeta, \xi))=\langle\xi, \Upsilon(\xi), \hbar(\zeta, \xi)\rangle$, problem (2.1) is called variational-like inequality with trifunction of finding $\xi \in \mathbb{K}_{\hbar}$, such that

$$
\langle\xi, \Upsilon(\xi), \hbar(\zeta, \xi)\rangle \geq 0, \forall \zeta \in \mathbb{K}_{\hbar}
$$

For $\langle\xi, \Upsilon(\xi), \hbar(\zeta, \xi)\rangle=\langle\Upsilon(\xi), \hbar(\zeta, \xi)\rangle$, problem (2.2) is called variational-like inequality of finding $\xi \in \tilde{H}$ such that

$$
\langle\Upsilon(\xi), \hbar(\zeta, \xi)\rangle \geq 0, \forall \zeta \in \mathbb{K}_{\hbar}
$$

If $\hbar(\zeta, \xi)=\zeta-\xi$, then an invex set reduces to a convex set and problem (2.1) is equivalent to finding $\xi \in \mathbb{K}$, such that

$$
\psi(\xi, \Upsilon(\xi), \zeta-\xi) \geq 0, \forall \zeta \in \mathbb{K}
$$


which is called an equilibrium problem with trifunction and gives off an impression of being new. Variational-like inequality with trifunction (2.2) reduces to variational inequality with trifunction of finding $\xi \in \mathbb{K}$, such that

$$
\langle\xi, \Upsilon(\xi), \zeta-\xi\rangle \geq 0, \forall \zeta \in \mathbb{K}
$$

Also variational-like inequality (2.3) is equivalent to finding $\xi \in \mathbb{K}$, such that

$$
\langle\Upsilon(\xi), \zeta-\xi\rangle \geq 0, \forall \zeta \in \mathbb{K}_{\hbar},
$$

which is known as variational inequality, proposed and cosidered by Stampacchia [20]. In brief, for suitable and appropriate choice of the functions $\Psi(., \ldots), \hbar(.,$.$) and the spaces, one can obtain a$ number of new and known problems as special cases of the problem (2.1). This shows that problem (2.1) is quite general and unifying.

Definition 2.5. The operator $\Upsilon: \tilde{H} \rightarrow \Re$ and the function $\Psi(., .,$.$) are said to be:$

(i) jointly $\hbar-$ pseudomonotone, if

$$
\begin{gathered}
\Psi(\xi, \Upsilon(\xi), \hbar(\zeta, \xi)) \geq 0 \\
\Longrightarrow \Psi(\zeta, \Upsilon(\zeta), \hbar(\xi, \zeta)) \leq 0, \forall \xi, \zeta \in \mathbb{K}_{\hbar} .
\end{gathered}
$$

(ii) partially relaxed jointly strong $\hbar-$ monotone, if there exists a constant $\alpha>0$ such that

$$
\begin{array}{r}
\Psi(\xi, \Upsilon(\xi), \hbar(\zeta, \mathfrak{z}))+\Psi(\zeta, \Upsilon(\zeta), \hbar(\mathfrak{z}, \zeta)) \leq \alpha\|\hbar(\mathfrak{z}, \xi)\|^{2} \\
\forall \xi, \zeta, \mathfrak{z} \in \mathbb{K}_{\hbar} .
\end{array}
$$

(iii) jointly $\hbar-$ monotone, if

$$
\Psi(\xi, \Upsilon(\xi), \hbar(\zeta, \xi))+\Psi(\zeta, \Upsilon(\zeta), \hbar(\xi, \zeta)) \leq 0, \forall \xi, \zeta \in \mathbb{K}_{\hbar}
$$

(iv) jointly $\hbar-$ hemicontinuous, if the mapping

$$
\Psi(\xi+\tau \hbar(\zeta, \xi), \Upsilon(\xi+\tau \hbar(\zeta, \xi)), \hbar(\zeta, \xi)) \forall \xi, \zeta \in \mathbb{K}_{\hbar}, \tau \in[0,1]
$$

is continuous. We note that, for $\mathfrak{z}=\xi$, partially relaxed jointly strong $\hbar-$ monotonicity reverts to jointly $\hbar-$ monotonicity.

Lemma 2.1. Let the trifunction $\Psi(., .,$.$) and operator \Upsilon$ be jointly $\hbar-$ pseudomonotone and jointly $\hbar$ - hemicontinuous. If Assumption (2.4) holds, then problem (2.1) is equivalent to finding $\xi \in \mathbb{K}_{\hbar}$, such that

$$
\Psi(\zeta, \Upsilon(\zeta), \hbar(\xi, \zeta)) \leq 0, \forall \xi, \zeta \in \mathbb{K}_{\hbar}
$$


Proof: Let $\xi \in \mathbb{K}_{\hbar}$ be a solution of equilibrium-like problem (2.1), then

$$
\Psi(\xi, \Upsilon(\xi), \hbar(\zeta, \xi)) \geq 0
$$

implies

$$
\Psi(\zeta, \Upsilon(\zeta), \hbar(\xi, \zeta)) \leq 0, \forall \xi, \zeta \in \mathbb{K}_{\hbar}
$$

since $\psi(., .,$.$) and \Upsilon$ are jointly $\hbar-$ pseudomonotone.

Conversely, let $\forall \xi, \zeta \in \mathbb{K}_{\hbar}$, we define $\zeta_{\tau}=\xi+\tau \hbar(\zeta, \xi) \in \tilde{H}$. Replacing $\zeta$ by $\zeta_{\tau}$ in (2.7), we have

$$
\Psi\left(\zeta_{\tau}, \Upsilon\left(\zeta_{\tau}\right), \hbar\left(\xi, \zeta_{\tau}\right)\right) \leq 0, \forall \xi, \zeta \in \mathbb{K}_{\hbar}
$$

Now by using Assumption (2.4), we have

$$
-\tau \Psi\left(\zeta_{\tau}, \Upsilon\left(\zeta_{\tau}\right), \hbar(\zeta, \xi)\right) \leq 0
$$

Now dividing (2.8) by $\tau$ and letting $\tau \longrightarrow 0$ and using jointly $\hbar-$ hemicontinuity of $\psi(., .,$.$) and \Upsilon$, we get

$$
\Psi(\xi, \Upsilon(\xi), \hbar(\zeta, \xi)) \geq 0
$$

which shows that $\xi \in \mathbb{K}_{\hbar}$ is a solution of problem (2.1), the required result.

Lemma (2.1) can be considered as the generalized form of Minty's Lemma for triequilibrium-like problems. Result obtained in above Lemma is termed as the dual triequilibrium -like problem.

\section{Well-Posedness}

We generalize the notion of well-posedness to triequilibrium-like problems. By using the assumption of pseudomonotonicity we obtain some results for well-posed equilibrium-like problems with trifunction. Our obtained results could be considered, an extension of the results which were obtained and studied in $[6,8,9,16-18]$

Given $\varepsilon>0$, consider two sets

$$
M(\varepsilon)=\left\{\xi \in \tilde{H}: \Psi(\xi, \Upsilon(\xi), \hbar(\zeta, \xi)) \geq-\varepsilon\|\hbar(\zeta, \xi)\|, \forall \zeta \in \mathbb{K}_{\hbar}\right\},
$$

and

$$
N(\varepsilon)=\left\{\xi \in \tilde{H}: \Psi(\zeta, \Upsilon(\zeta), \hbar(\xi, \zeta)) \leq \varepsilon\|\hbar(\zeta, \xi)\|, \forall \zeta \in \mathbb{K}_{\hbar} .\right.
$$

For a non-empty set $S \subset \tilde{H}$, define the diameter of $S$, denoted by $D(S)$, as:

$$
D(S)=\sup [\|\zeta-\xi\| ; \forall \xi, \zeta \in S] .
$$

Definition 3.1. [18]. The triequilibrium-like problem (2.1) is well-posed, if

(i) For any $\varepsilon>0, M(\varepsilon) \neq \emptyset$,

(ii) $D(M(\varepsilon)) \longrightarrow 0$ as $\varepsilon \longrightarrow 0$. 
Theorem 3.1. Let the trifunction $\Psi(., .,$.$) and operator \Upsilon$ be jointly $\hbar-$ pseudomonotone and jointly $\hbar$ - hemicontinuous. If Assumption (2.4) holds, then

$$
M(\varepsilon)=N(\varepsilon) .
$$

Proof: Let $\xi \in \mathbb{K}_{\hbar}$ be such that

$$
\Psi(\xi, \Upsilon(\xi), \hbar(\zeta, \xi)) \geq-\varepsilon\|\hbar(\zeta, \xi)\|, \forall \zeta \in \mathbb{K}_{\hbar}
$$

implies

$$
\Psi(\zeta, \Upsilon(\zeta), \hbar(\xi, \zeta)) \leq \varepsilon\|\hbar(\zeta, \xi)\|, \forall \zeta \in \mathbb{K}_{\hbar}
$$

since $\psi(., .,$.$) and \Upsilon$ are jointly $\hbar-$ pseudomonotone. Thus

$$
M(\varepsilon) \subset N(\varepsilon)
$$

Conversely, for any $\xi \in \mathbb{K}_{\hbar}$, (3.3) holds.

Now, let $\forall \xi, \zeta \in \mathbb{K}_{\hbar}$ we define $\zeta_{\tau}=\xi+\tau \hbar(\zeta, \xi) \in \mathbb{K}_{\hbar}$. Replacing $\zeta$ by $\zeta_{\tau}$ in (3.3), we have

$$
\Psi\left(\zeta_{\tau}, \Upsilon\left(\zeta_{\tau}\right), \hbar\left(\xi, \zeta_{\tau}\right)\right) \leq \varepsilon\left\|\hbar\left(\zeta_{\tau}, \xi\right)\right\|, \forall \xi, \zeta \in \mathbb{K}_{\hbar}
$$

Now by using Assumption (2.4), we have

$$
-\tau \Psi\left(\zeta_{\tau}, \Upsilon\left(\zeta_{\tau}\right), \hbar(\zeta, \xi)\right) \leq \tau \varepsilon\|\hbar(\zeta, \xi)\|
$$

Now dividing (3.5) by $\tau$ and letting $\tau \longrightarrow 0$ and using jointly $\hbar-$ hemicontinuity of $\Psi(., .,$.$) and T$, we get

$$
\Psi(\xi, \Upsilon(\xi), \hbar(\zeta, \xi)) \geq-\varepsilon\|\hbar(\zeta, \xi)\|
$$

which implies that

$$
N(\varepsilon) \subset M(\varepsilon)
$$

By combining (3.4) and (3.6), we get our required result

$$
N(\varepsilon)=M(\varepsilon)
$$

Theorem 3.2. Let the trifunction $\Psi(., .,$.$) and operator \Upsilon$ be jointly $\hbar-$ pseudomonotone and jointly $\hbar$ - hemicontinuous. Then for all $\varepsilon>0, N(\varepsilon)$ is closed in $\tilde{H}$.

Proof: Let $\left\{\xi_{n}: n \in \mathcal{N}\right\} \subset N(\varepsilon)$ be a sequence, such that $\xi_{n} \longrightarrow \xi$ in $\mathbb{K}_{\hbar}$ as $n \longrightarrow \infty$. Then $\xi_{n} \in \mathbb{K}_{\hbar}$ and

$$
\Psi\left(\zeta, \Upsilon(\zeta), \hbar\left(\xi_{n}, \zeta\right)\right) \leq \varepsilon\left\|\hbar\left(\zeta, \xi_{n}\right)\right\|, \forall \zeta \in \mathbb{K}_{\hbar}
$$

By taking limit $n \longrightarrow \infty$ in (3.7), we get

$$
\psi(\zeta, \Upsilon(\zeta), \hbar(\xi, \zeta)) \leq \varepsilon\|\hbar(\zeta, \xi)\|, \forall \zeta \in \mathbb{K}_{\hbar}
$$


which implies that $\xi \in N(\varepsilon)$. Thus, $N(\varepsilon)$ is closed in $\mathbb{K}_{\hbar}$

Theorem 3.3. Let the trifunction $\Psi(., .,$.$) and operator \Upsilon$ be jointly $\hbar-$ pseudomonotone and jointly $\hbar$ - hemicontinuous. If triequilibrium-like problem (2.1) is well-posed and Assumption (2.4) holds, then there exists a unique solution of problem (2.1).

Proof: Let the sequence $\left\{\xi_{n}: n \in \mathcal{N}\right\}$, defined by $\xi_{n} \in M\left(\frac{1}{n}\right)$. Let $\varepsilon>0$ be very small and let $p, q \in \mathcal{N}$, such that $p \geq q \geq \frac{1}{\varepsilon}$. Then

$$
M\left(\frac{1}{p}\right) \subset M\left(\frac{1}{q}\right) \subset M(\varepsilon)
$$

So

$$
\left\|\xi_{p}-\xi_{q}\right\| \leq D\left(M\left(\frac{1}{p}\right)\right)
$$

Thus, the sequence $\left\{\xi_{n}\right\}$ is a Cauchy sequence and $\xi_{n} \longrightarrow \xi$ in $\mathbb{K}_{\hbar}$. By using results of Theorem (3.1) and Theorem (3.2), we get $M(\varepsilon)$ is a closed set. Thus

$$
\xi \in \bigcap_{\varepsilon>0} M(\varepsilon)
$$

so, $\xi$ is solution of problem (2.1). Uniqueness of solution $\xi$ follows from second condition of wellposedness.

Theorem 3.4. Let the trifunction $\Psi(., .,$.$) and operator \Upsilon$ be jointly $\hbar-$ pseudomonotone and jointly $\hbar$ - hemicontinuous. If $M(\varepsilon) \neq 0$, for all $\varepsilon>0$ and $M(\varepsilon)$ is bounded for some $\varepsilon_{0}$, then there exists at least one solution of problem (2.1).

Proof: Let $\xi_{k} \in M\left(\frac{1}{k}\right)$, then for large enough $k$, we have

$$
M\left(\frac{1}{k}\right) \subset M(\varepsilon) .
$$

Thus for some subsequence $\xi_{k} \longrightarrow \xi \in \mathbb{K}_{\hbar}$, we get

$$
\begin{aligned}
\psi\left(\zeta, \Upsilon(\zeta), \hbar\left(\xi_{k}, \zeta\right)\right) & \leq \frac{1}{k}\left\|\hbar\left(\zeta, \xi_{k}\right)\right\| \\
& \leq \frac{1}{k}\{\|\zeta\|+c\} \forall \in \tilde{H} .
\end{aligned}
$$

Now by taking limit $k \longrightarrow \infty$, we get

$$
\Psi(\zeta, \Upsilon(\zeta), \hbar(\xi, \zeta)) \leq 0
$$

which implies that $\xi \in N(0)$. By Theorem (3.1), we get $\xi \in N(0)=M(0)$, which implies $\xi \in M(0)$. Hence triequilibrium-like problem (2.1) has at least one solution. 
Remark 3.1. (i) If triequilibrium-like problem has a unique solution, then $M(\varepsilon) \neq 0, \forall \varepsilon>0$ and $\bigcap_{\varepsilon>0}=\left\{\xi_{0}\right\}$.

(ii) It is well-known, that if there is a unique solution to variational inequality (2.6), then it is not well-posed, see [9].

(iii) Theorem (3.3) concludes that, the unique solution of problem (2.1) could be calculated by utilizing $\varepsilon$ - equilibrium-like problem with trifunction, that is, find $\xi_{\varepsilon} \in \tilde{H}$, such that

$$
\Psi\left(\xi_{\varepsilon}, \Upsilon\left(\xi_{\varepsilon)}, \hbar\left(\zeta, \xi_{\varepsilon}\right)\right) \geq-\varepsilon\left\|\hbar\left(\zeta, \xi_{\varepsilon}\right)\right\|, \forall \zeta \in \mathbb{K}_{\hbar}\right.
$$

\section{Perspective}

In this section, we discuss some research perspective of the equilibrium-like problem with trifunction. We show that the results derived in this paper can also be extended for a class of nonconvex equilibrium problem with trifunction.

For the given operators $\Upsilon, g: \tilde{H} \rightarrow \Re$ and a nonlinear continuous trifunction $\Psi(., \ldots): \tilde{H} \times \tilde{H} \times \tilde{H} \rightarrow \Re$, consider the problem of finding $\xi \in \mathbb{K}_{\hbar}$, such that

$$
\Psi\left(g(\xi), \Upsilon(g(\xi)),(g(\zeta)) \geq 0, \forall \zeta \in \mathbb{K}_{\hbar},\right.
$$

which is called nonconvex triequilibrium problem. For the formulation and applications of problem (4.1) see, $[12,15,18]$ and references therein. For $g \equiv I$, where $/$ is the identity operator, then the $g_{-}$ convex set becomes becomes the convex set and the problem (4.1) is called the triequilibrium problem of finding $\xi \in \mathbb{K}_{\hbar}$, such that

$$
\Psi(\xi, \Upsilon(\xi), \zeta) \geq 0, \forall \zeta \in \mathbb{K}_{\hbar}
$$

which was introduced and investigated by Noor and Oettli [14]. For $\hbar(\zeta, \xi)=\zeta-\xi$, the invex set reduces to convex set. Thus from (4.1) and (4.2), we obtain that the triequilibrium-like problem is equivalent to the nonconvex equilibrium problem with trifunction (4.1). Hence all the above discussed results continue to hold for nonconvex triequilibrium problem (4.1).

\section{Conclusion}

In our work we focused on introducing another class of equilibrium-like problems terming it as triequilibrium-like problems. We studied and established the well-posedness of triequilibrium-like problems by using the assumption of pseudomonotonicity. The demonstrated results in this endeavour can rightly be considered as an enhancement and sophistication of already existing work.

\section{Acknowledgements}

We wish to express our deepest gratitude to our colleagues, collaborators and friends, who have direct or indirect contributions in the process of this paper. We are also grateful to Rector, COMSATS University Islamabad, Pakistan for the research facilities and support in our research endeavors. 
Conflicts of Interest: The author(s) declare that there are no conflicts of interest regarding the publication of this paper.

\section{References}

[1] A. Ben-Israel and B. Mond, What is invexity?, J. Austral. Math. Soc. Ser. B. 28 (1986), 1-9. https://doi.org/ 10.1017/S0334270000005142.

[2] M. I. Bloach and M. A. Noor, Perturbed mixed variational-like inequalities, AIMS Math. 5(3) (2019), $2153-2162$. https://doi.org/10.3934/math. 2020143.

[3] E. Blum and W. Oettli, From optimization and variational inequalities to equilibrium problems, Math. Student. 63 (1994), 123-145.

[4] R. Glowinski, J. L. Lions and R. Tremolieres, Numerical analysis of variational inequalities, North-Holland, Amsterdam, 1981.

[5] F. Giannessi and A. Maugeri, Variational Inequalities and Network Equilibrium Problems. Plenum Press, New York, 1995.

[6] F. Giannessi, A. Maugeri and P. M. Pardalos, Equilibrium Problems: Nonsmooth Optimization and Variational inequality Models, Kluwer Academic Publishers, Dordrecht, Holland, 2001.

[7] D. Goeleven and D. Mantaque, Well-posed hemivariational inequalities. Numer. Funct. Anal. Optim. 16 (1995), 909-921. https://doi.org/10.1080/01630569508816652.

[8] M. A. Hanson, On sufficiency of the Kuhn-Tucker conditions, J. Math. Anal. Appl. 80 (1981), 545-550. https: //doi.org/10.1016/0022-247X (81)90123-2.

[9] R. Lucchetti and F. Patrone, A characterization of Tykhonov well-posedness for minimum problems with applications to variational inequalities. Numer. Funct. Anal. Optim. 3 (1981), 461-476. https://doi.org/10.1080/ 01630568108816100.

[10] R. Lucchetti and F. Patrone, Some properties of well-posed variational inequalities governed by linear operators. Numer. Funct. Anal. Optim. 5 (1983), 349-361. https://doi.org/10.1080/01630568308816145.

[11] S. R. Mohan and S. K. Neogy, On invex set and preinvex functions. J. Math. Anal. Appl. 189 (1995), $901-908$. https://doi.org/10.1006/jmaa.1995.1057.

[12] T. V. Nghi1 and N. N. Tam, General variational inequalities: existence of solutions, Tikhonov-Type regularization, and well-posedness, Acta Math. Vietnam. (2021). https://doi.org/10.1007/s40306-021-00435-0.

[13] B. B. Mohsen, M. A. Noor, K. I. Noor and M. Postolache, Strongly convex functions of higher order involving bifunction, Mathematics, 7(11) (2019), 1028. https://doi.org/10.3390/math7111028.

[14] M. A. Noor, General variational inequalities. Appl. Math. Lett. 1 (1988), 119-121. https://doi.org/10.1016/ 0893-9659(88) 90054-7.

[15] M. A. Noor, Variational-like inequalities. Optimization, 30 (1994), 323-330. https://doi.org/10.1080/ 02331939408843995.

[16] M. A. Noor, New approximation schemes for general variational inequalities. J. Math. Anal. Appl. 251 (2000), 217-229. https://doi.org/10.1006/jmaa.2000.7042.

[17] M. A. Noor, Merit function for variational-like inequalitiets, Math. Inequal. Appl. 1 (2000), 117-128.

[18] M. A. Noor, Well-posed variational inequalities. J. Appl. Math. Comput. 11 (2003), 165-172. https://doi.org/ 10.1007/BF02935729.

[19] M. A. Noor, Fundamentals of mixed quasi variational inequalities. Int. J. Pure. Appl. Math. 15 (2004), $137-250$.

[20] M. A. Noor, Fundamentals of equilibrium problems. Math. Inequal. Appl. 9 (2006), 529-566. https://doi.org/ 10.7153/mia-09-51. 
[21] M. A. Noor, Extended general variational inequalities. Appl. Math. Lett. 22(2) (2009), 182-186. https://doi. org/10.1016/j.aml.2008.03.007.

[22] M. A. Noor and K. I. Noor, Some new trends in mixed variational inequalities, J. Adv. Math. Stud. in press.

[23] M. A. Noor and W. Oettli, On general nonlinear complementarity problems and quasi-equilibria. Le Mathematiche, 49 (1994), 313-331.

[24] M. A. Noor, K. I. Noor and H. M. Y. Al-Bayatti, Higher order variational inequalities, Inf. Sci. Lett. 11 (2022), 1-5.

[25] M. A. Noor, K. I. Noor and M. I. Baloch, Auxiliary principle technique for strongly mixed variational-like inequalities. U.P.B. Sci. Bull. Ser. A, 80 (2018), 93-100.

[26] M. A. Noor, K. I. Noor, A. Hamdi and E. H. El-shemas, On difference of two monotone operators, Optim. Lett. 3 (2009), 329. https://doi.org/10.1007/s11590-008-0112-7.

[27] M.A. Noor, K.I. Noor, M.Th. Rassias, New trends in general variational inequalities, Acta Appl. Math. 170 (2020), 981-1064. https://doi.org/10.1007/s10440-020-00366-2.

[28] M. A. Noor, K. I. Noor, M. U. Awan and A. G. Khan, Quasi variational inclusions involving three operators, Inform. Sci. Lett. in press.

[29] G. Stampacchia, Formes bilineaires coercitives sur les ensembles convexes. C. R. Acad. Sci. Paris, 258 (1964), 4413-4416.

[30] T. Weir and B. Mond, Preinvex functions in multiple objective optimization. J. Math. Anal. Appl. 136 (1988), 29-38. https://doi.org/10.1016/0022-247X (88)90113-8.

[31] T. Weir and V. Jeyakumar, A class of nonconvex functions and mathematical programming. Bull. Austral. Math. Soc. 38 (1988), 177-189. https://doi.org/10.1017/S0004972700027441. 